\title{
AN EXAMPLE OF A WEIGHT WITH TYPE III CENTRALIZER
}

\author{
UFFE HAAGERUP
}

\begin{abstract}
We give an example of a weight $\phi$ on the hyperfinite type $\mathrm{II}_{\infty}$ factor $R$, such that the centralizer $M_{\phi}$ of $\phi$ is a type III von Neumann algebra.
\end{abstract}

Introduction. Let $\phi$ be a normal, semifinite, faithful weight on a von Neumann algebra $M$, and $\sigma_{t}^{\phi}$ the modular automorphism group associated with $\phi$. The centralizer $M_{\phi}$ is the fixed point-algebra under $\sigma_{t}^{\phi}$, i.e.,

$$
M_{\phi}=\left\{x \in M \mid \sigma_{t}^{\phi}(x)=x, t \in R\right\} .
$$

The restriction of $\phi$ to $M_{\phi}$ is a trace. Hence if $\phi$ is strictly semifinite (i.e. the restriction of $\phi$ to $M_{\phi}$ is semifinite), then $M_{\phi}$ is a semifinite von Neumann algebra (cf. [1]). In particular, if $\phi$ is bounded, then $M_{\phi}$ is finite. Recently A. Connes and $M$. Takesaki have proved that $M_{\phi}$ is semifinite for a great class of non strictly semifinite weights, namely the integrable weights, i.e. those weights $\phi$, for which the set $\left\{x \in M_{+} \mid \int_{-\infty}^{\infty} \sigma_{t}^{\phi}(x) d t \in M_{+}\right\}$is $\sigma$-weakly dense in $M_{+}$(cf. [3]).

In this paper we prove that $M_{\phi}$ is not, in general, semifinite. At the same time we find that the relative commutant $N^{\prime} \cap M$ of a semifinite von Neumann algebra $N$ in a semifinite von Neumann algebra $M$ is not, in general, semifinite (Lemma 2). That is the negative solution to a problem considered in [2].

LEMMA 1. On a separable Hilbert space $H$, there exists a hyperfinite type $\mathrm{II}_{1}$ factor $M$ and an abelian von Neumann algebra $A \subseteq M^{\prime}$, such that the von Neumann algebra $(M \cup A)^{\prime \prime}$ is of type III.

Proof. Let $B=\bigotimes_{n=1}^{\infty} F_{n}$ be the uniformly hyperfinite $C^{*}$-algebra obtained as tensor product of a sequence of type $I_{2}$-factors (cf. [5, Chapter 1, §23]). B has a unique normalized trace $\tau$, and $\pi_{\tau}(B)^{\prime \prime}$ is the hyperfinite type $\mathrm{II}_{1}$-factor. Let $U_{n}$ be the unitary group in $F_{n}$, and put $G=\prod_{n=1}^{\infty} U_{n}$. Then $G$ is a compact group with normalized Haar measure $d g$. We define an action of $G$ as *-automorphisms on $B$ in the following way: For $g \in G, g=\left(u_{n}\right)_{n \in N}$, we put

$$
\sigma_{g}=\bigotimes_{n=1}^{\infty} \operatorname{ad}\left(u_{n}\right)
$$

Received by the editors February 2, 1976.

AMS (MOS) subject classifications (1970). Primary 46L10.

Key words and phrases. von Neumann algebras, Tomita-Takesaki theory, weights.

@ American Mathematical Society 1977 
It is easy to check that $g \rightarrow \sigma_{g}$ is a homeomorphism, and that $(g, x) \rightarrow \sigma_{g} x$ is jointly continuous. Let $\phi$ be a state on $B$, and put

$$
\omega(x)=\int_{G} \phi \cdot \sigma_{g}(x) d g .
$$

Let $\omega_{n}$ (resp. $\tau_{n}$ ) be the restriction of $\omega$ (resp. $\tau$ ) to $F_{1} \otimes F_{2} \otimes \cdots \otimes F_{n}$. By definition $\omega_{n}$ is invariant under $\operatorname{ad}\left(u_{1}\right) \otimes \cdots \otimes \operatorname{ad}\left(u_{n}\right), u_{k} \in U_{k}, k=$ $1, \ldots, n$. From this it follows that $\omega_{n}=\tau_{n}$ for any $n \in N$, and thus $\omega=\tau$. Hence for any state $\phi$ on $B$,

$$
\tau(x)=\int_{G} \phi \cdot \sigma_{g}(x) d g .
$$

In the following we let $\phi$ be a fixed type III state on $B$. On the $C^{*}$-tensor product $B \otimes C(G)=C(G, B)$ we regard the state

$$
\tilde{\phi}(x(g))=\int_{G} \phi \cdot \sigma_{g}(x(g)) d g, \quad x \in B \otimes C(G) .
$$

$\tilde{\phi}$ is a type III state, because

$$
\tilde{\phi}(x)=(\phi \otimes d g) \tilde{\sigma}(x), \quad x \in B \otimes C(G),
$$

where $\tilde{\sigma}$ is the *-automorphism of $B \otimes C(G)$ given by $\tilde{\sigma}(x)(g)=\sigma_{g} x(g)$. Hence $N=\pi_{\tilde{\phi}}(B \otimes C(G))^{\prime \prime}$ is of type III. However, $N$ is generated by $\pi_{\tilde{\pi}}(B \otimes I)^{\prime \prime}$ and $\pi_{\tilde{\phi}}(I \otimes C(G))^{\prime \prime}$. The first is isomorphic to the hyperfinite type $\mathrm{II}_{1}$-factor $\pi_{\tau}(B)^{\prime \prime}$ because $\tilde{\phi}(x \otimes I)=\tau(x), x \in B$, and the latter is commutative. Moreover the two algebras commute. Since $B \otimes C(G)$ is a separable $C^{*}$-algebra, the Hilbert space $H_{\tilde{\phi}}$ associated with $\pi_{\tilde{\phi}}$ is separable. This completes the proof.

Lemma 2. Let $R$ be the hyperfinite factor of type $\mathrm{II}_{\infty}$. There exists an abelian sub von Neumann algebra $A \subseteq R$, such that the relative commutant $R \cap A^{\prime}$ is of type III.

Proof. Let $M$ and $A$ be as in Lemma 1. Put $R=M^{\prime}$. Then $R \cap A^{\prime}=$ $(M \cup A)^{\prime}$ is of type III. Since $R$ contains a type III von Neumann algebra, it is of infinite multiplicity. Hence $R \simeq M \otimes F_{\infty}$ where $F_{\infty}$ is a type $\mathrm{I}_{\infty}$ factor.

THEOREM. There exists a normal, semifinite faithful weight on the hyperfinite factor of type $\mathrm{II}_{\infty}$, such that the centralizer $M_{\phi}$ is of type III.

Proof. Let $A$ and $R$ be as in Lemma 2. Since $A$ is an abelian von Neumann algebra, which can be represented faithfully on a separable Hilbert space, $A$ is the von Neumann algebra generated by a single selfadjoint element $h$. We may assume that $h \geqslant 1$. Put $\phi=\tau(h \cdot)$ where $\tau$ is the trace on $R$. Then

$$
\sigma_{i}^{\phi}(x)=h^{i t} x h^{-i t}, \quad x \in R \quad \text { (cf. [4]). }
$$

Hence $M_{\phi}=R \cap\{h\}^{\prime}=R \cap A^{\prime}$ which is of type III. 


\section{REFERENCES}

1. F. Combes, Poids et espérances conditionnelles dans les algèbres de von Neumann, Bull. Soc. Math. France 99 (1971), 73-112. MR 44 \# 5785.

2. F. Combes and $\mathrm{C}$. Delaroche, Groupe modulaire d'une espérance conditionnelle dans une algèbre de von Neumann, Université d'Orléans, 1974 (preprint).

3. A. Connes and M. Takesake, Flow of weights on factors of type III, 1975 (preprint).

4. G. K. Pedersen and M. Takesaki, The Radon-Nikoy'm theorem for von Neumann algebras, Acta Math. 130 (1973), 53-87.

5. Shôichirô Sakai, $C^{*}$-algebras and $W^{*}$-algebras, Springer-Verlag, Berlin, 1971.

Odense Universitet, Odense, Denmark 